\title{
MID-IR SPECTRA OF AGB AND POST-AGB STARS
}

\author{
C. J. SKINNER \\ Institute of Geophysics \& Planetary Physics, L-413, Lawrence Livermore National \\ Laboratory, P.O.Box 808, Livermore, CA94551-9900, U.S.A. \\ and \\ M. J. BARLOW, K. JUSTTANONT and R. J. SYLVESTER \\ Dept. of Physics \& Astronomy, University College London, Gower Street, London WC1E \\ $6 B T$, Great Britain
}

We have generated models of complete nova energy distributions, including the effects of the nova dust shell emission and absorption. In order to account for the dust, we require optical constants throughout the UV and EUV. Draine \& Lee (1984) is the only source of such data for silicates. However, their 'astronomical silicate' has a strong resonance in the UV which causes circumnova dust to be heated far above its sublimation temperature in our models of Nova Her 1991. We have therefore recalculated the silicate optical constants, using the KramersKronig relations, including a $\lambda^{-1.0}$ far-IR dependence and improved $9.7 \mu \mathrm{m}$ feature, and decreasing the UV and EUV efficiencies of the grains. With these new silicate constants we can reproduce the observed behaviour of the dust shell in Nova Her 1991. Since our optical constants are consistent with the behaviour of Nova Her 1991, an EUV source, they should also be appropriate for the treatment of PN.

The $10 \mu \mathrm{m}$ silicate feature in Nova Her is most unusual. Instead of peaking near $9.7 \mu \mathrm{m}$, as in most O-rich AGB stars, the feature is very broad with a flat top, extending from $\sim 9.7 \mu \mathrm{m}$ to $11.5 \mu \mathrm{m}$. We observe such behaviour in silicate features in several PPN. In the case of AFGL2343 and HD161796 we ascribed the broad, rather flat silicate profiles to their cool dust shells (Justannont et al. , 1992). A third post-AGB source, OH53.8+20.2, demonstrates this behaviour particularly strongly. Contrarily, a fourth post-AGB star of the same type, V1027 Cyg, shows a remarkably strong $9.7 \mu \mathrm{m}$ silicate feature which strongly resembles that seen in AGB stars, typified by the strong feature in the well known M-Supergiant $\mu$ Cep. The three post-AGB sources with unusual silicate profiles, AFGL2343, HD161796 and $\mathrm{OH} 53.8+20.2$, all have rather cool dust shells, indicating that these stars left the AGB long ago. On the other hand V1027 Cyg has a much hotter energy distribution, indicating that it left the AGB only recently. We suggest that the unusual silicate profiles have arisen because the warm central stars have annealed the dust in the time since these stars left the AGB. Similarly, in Nova Her 1991 the dust forms in and is exposed continuously to an exceptionally harsh radiation field.

\section{References}

Draine, B. T., Lee, H-M.: 1984, 'Optical Properties of Interstellar Graphite and Silicate Grains', Ap. J. 285, 89

Justtanont, K., Barlow, M. J., Skinner, C. J., Tielens, A. G. G. M.: 1992, 'The Nature of Dust around the Post-asymptotic Giant Branch Objects HD161796 and HD179821', Ap. J. 392, L75 